\title{
Age-related Effects on Word Recognition: Reliance on Cognitive Control Systems with Structural Declines in Speech-responsive Cortex
}

\author{
Mark A. Eckert, Adam Walczak, Jayne Ahlstrom, Stewart Denslow, Amy Horwitz, \\ AND Judy R. Dubno \\ ${ }^{1}$ Department of Otolaryngology-Head and Neck Surgery, Medical University of South Carolina, 135 Rutledge Avenue, MSC \\ 550, Charleston, SC 29425-5500, USA
}

Received: 29 October 2007; Accepted: 16 January 2008; Online publication: 15 February 2008

\begin{abstract}
Speech recognition can be difficult and effortful for older adults, even for those with normal hearing. Declining frontal lobe cognitive control has been hypothesized to cause age-related speech recognition problems. This study examined age-related changes in frontal lobe function for 15 clinically normal hearing adults (21-75 years) when they performed a word recognition task that was made challenging by decreasing word intelligibility. Although there were no age-related changes in word recognition, there were age-related changes in the degree of activity within left middle frontal gyrus (MFG) and anterior cingulate (ACC) regions during word recognition. Older adults engaged left MFG and ACC regions when words were most intelligible compared to younger adults who engaged these regions when words were least intelligible. Declining gray matter volume within temporal lobe regions responsive to word intelligibility significantly predicted left MFG activity, even after controlling for total gray matter volume, suggesting that declining structural integrity of brain regions responsive to speech leads to the recruitment of frontal regions when words are easily understood.
\end{abstract}

Electronic supplementary material The online version of this article (doi:10.1007/s10162-008-0113-3) contains supplementary material, which is available to authorized users.

Correspondence to: Mark A. Eckert - Department of OtolaryngologyHead and Neck Surgery $\cdot$ Medical University of South Carolina $\cdot 135$ Rutledge Avenue, MSC 550, Charleston, SC 29425-5500, USA. Telephone: +1-843-7925914; fax: +1-843-7927736; email: eckert@ musc.edu
Keywords: aging, word recognition, speech, attention, fMRI, voxel-based morphometry, middle frontal gyrus, anterior cingulate, superior temporal gyrus

\section{INTRODUCTION}

Speech recognition becomes progressively more difficult and effortful with age. While hearing loss in older adults is a primary contributing factor, age-related declines in speech recognition are observed independently of hearing loss (van Rooij and Plomp 1990; Dubno et al. 1997). Speech recognition is particularly affected in complex and demanding listening conditions in which word intelligibility is made difficult (Sommers and Danielson 1999; Gordon-Salant and Fitzgibbons 2001, 2004; Dubno et al. 2005, 2006). Neural systems that support cognitive control become increasingly engaged in demanding listening conditions (Obleser et al. 2007). A failure of cognitive control, specifically the failure to inhibit irrelevant stimuli and focus on speech, has been proposed to explain the speech recognition difficulties of older adults (Sommers 1997; Dywan et al. 2001) and cognitive declines in general (Gazzaley and D'Esposito 2007).

The frontal lobe systems that support cognitive control demonstrate age-related structural and functional changes (Raz et al. 1997; Milham et al. 2002; Nielson et al. 2002; Cabeza et al. 2004; Tisserand et al. 2004; Colcombe et al. 2005). In particular, the ACC and MFG exhibit age-related changes in activation during speech comprehension and memory retrieval tasks (Grady et al. 2005; Sharp et al. 2006). The 
findings from many age-related imaging studies indicate that older adults exhibit increased frontal lobe activity during memory (Cabeza et al. 1997; McIntosh et al. 1999; Reuter-Lorenz et al. 2000; Rypma and D'Esposito 2000; Cabeza et al. 2002), perception (Grady et al. 1994; Fernandes et al. 2006; Moffat et al. 2006), and response inhibition tasks (Milham et al. 2002; Nielson et al. 2002). This increased frontal activity is hypothesized: (1) to be compensatory for high functioning older adults (Cabeza et al. 2002), (2) to reflect the need for greater cognitive control than younger adults (Dywan et al. 2002), and (3) to reflect the increased amount of irrelevant information that older adults retain in working memory compared to younger adults (Hasher and May 1999). While one study has examined age-related changes in speech comprehension (Sharp et al. 2006), there are no imaging studies that have examined age-related changes in word recognition. This study examined the extent to which frontal lobe regions exhibited age-related changes in activity during word recognition when words were filtered to parametrically vary word intelligibility. In addition, we hypothesized that structural declines in the temporal lobe regions that are responsive to speech would predict the increased reliance on frontal lobe systems for word recognition.

\section{MATERIALS AND METHODS}

\section{Subjects}

Fifteen adults, ranging in age from 21-75 years (mean 42.1, SD 18.7 years; nine female), participated in this study. The participants were recruited from the Medical University of South Carolina (MUSC) community and Charleston, SC area through word of mouth and a longitudinal study of age-related hearing loss (presbyacusis). Their average years of education was 17.7, SD 2.0 (16 years is equivalent to a 4 -year college degree). The aims of this study were explained to each participant and MUSC Institutional Review Board-approved informed consent was obtained. All participants in this study had audiometric thresholds below $25 \mathrm{~dB} \mathrm{HL}$ at octave frequencies from 250 to $3000 \mathrm{~Hz}$ (ANSI 2004). In addition, threshold masking noise (described below) was used to control for individual differences in hearing thresholds below $25 \mathrm{~dB}$ HL.

\section{Image acquisition and task design}

A sparse sampling design was used to: (1) limit the confounding influence of scanner noise on the stimuli and on neural responses to the stimuli; (2) provide time to generate a verbal response; and (3) provide time for participants to stabilize their heads before the next TR (Fridriksson et al. 2006). T2*weighted functional images were acquired on a Philips 3T scanner using a single shot echo-planar imaging (EPI) sequence that covers the whole brain (32 slices with a $64 \times 64$ matrix, $\mathrm{TR}=8 \mathrm{~s}, \mathrm{TE}=30 \mathrm{~ms}$, slice thickness $=3.25 \mathrm{~mm}$, and a $\mathrm{TA}=1,647 \mathrm{~ms}$ ). One volume was collected for each $8 \mathrm{~s}$ TR. T1-weighted images were also collected for brain structure analyses (160 slices with a $256 \times 256$ matrix, $\mathrm{TR}=8.13 \mathrm{~ms}, \mathrm{TE}=$ $3.7 \mathrm{~ms}$, flip angle $=8^{\circ}$, slice thickness $=1 \mathrm{~mm}$, and no slice gap).

Each participant performed a word recognition task in which they listened to 40 words that were presented across four randomly ordered low-pass filter conditions for a total of 160 event-related trials (upper cutoff frequencies $=400,1,000,1,600$, and $3,150 \mathrm{~Hz}$; the lower cutoff frequency was fixed at $200 \mathrm{~Hz}$; audio examples are presented in Supplementary Materials). We chose to filter the words to vary word intelligibility rather than use multi-talker babble or noise-vocoded stimuli as a preliminary step towards understanding cortical representation for speech in people with high frequency hearing loss, which will be addressed in future studies. The words were nouns selected from a list of 400 monosyllabic consonantvowel-consonant words used by Dirks et al. (2001) and presented at $75 \mathrm{~dB}$ SPL. Lexical difficulty of the words [the combined influence of word frequency (mean $=43.2, \mathrm{SD}=77.4)$, the number of other similarsounding words (mean=18.3, $\mathrm{SD}=6.6$ ), and the frequency of the similar sounding words (mean $=243.5$, $\mathrm{SD}=444.1$ ), Sommers and Danielson 1999] was normally distributed. One goal of this study was to examine age-related changes in central representations for speech while controlling for peripheral auditory system factors that might affect individual variability in cortical responsiveness to speech. Towards that end, we included only adults with clinically normal hearing and we presented a broadband masker across each $8 \mathrm{~s}$ TR to minimize individual differences in hearing thresholds. The broadband masker was digitally generated and its spectrum was adjusted at one-third-octave intervals to produce equivalent masked thresholds for all subjects, and thus control for individual differences in hearing thresholds. Band levels of the noise were set to achieve masked thresholds of 20-25 dB HL from 200 to $3,150 \mathrm{~Hz}, 30 \mathrm{~dB} \mathrm{HL}$ at 4,000 and $5,000 \mathrm{~Hz}$, and $40 \mathrm{~dB} \mathrm{HL}$ at $6,300 \mathrm{~Hz}$. Pilot testing of the word recognition experiment was performed with 10 young adults in a double-walled sound-treated room, which demonstrated that word recognition decreased linearly with decreased cutoff frequency $(r=0.99)$ without a ceiling effect for the $3,150-\mathrm{Hz}$ condition. Nearly identical word recognition results were observed in 
the experimental sample of 15 subjects (ages 21-75) measured during MR scanning. Eprime software (Psychology Software Tools Inc.) and an IFIS-SA control system (Invivo Corp.) were used to present the words to subjects in the scanner. The broadband noise was presented from a separate PC throughout the experiment. The words were mixed with the broadband noise at precisely $2.5 \mathrm{~s}$ into the $8 \mathrm{~s}$ TR using a standard audio mixer, and then delivered to the subject through custom-made piezoelectric insert earphones (Sensimetrics Corp.). Signal levels were calibrated using a Bruel \& Kjaer sound-level meter (Type 2231). Participants were instructed to listen and respond with the word they heard or with "nope" if they could not recognize the word, ensuring that a motor response was produced on each trial. Each response was recorded as correct, incorrect, or "nope" by two raters (M.E. and A.W.). An overt oral response was chosen so that the results were directly relatable to audiologic assessment of word recognition and because speech production tasks have been used successfully in other language studies (Gracco et al. 2005; Shuster and Lemieux 2005; Fridriksson et al. 2006). Figure 1 summarizes the experimental design used in this study.

\section{Data analyses}

Image pre-processing was performed using SPM5 algorithms (http://www.fil.ion.ucl.ac.uk/spm). Each participant's native space images were realigned to the first volume and unwarped to correct for head movement and susceptibility distortions. Image volumes, slices, and voxels with significant artifact were identified using the ArtRepair toolbox (http://cibsr. stanford.edu/tools/ArtRepair/ArtRepair.htm) based on scan-to-scan motion (1 SD change in head position) and outliers relative to the global mean signal (3 SD from the global mean). An average of three image volumes (SD 1.6) was excluded for artifact from each subject's dataset. The images were normalized to the ICBM EPI template and smoothed with an 8-mm Gaussian kernel to ensure that the data were normally distributed and appropriate for para-

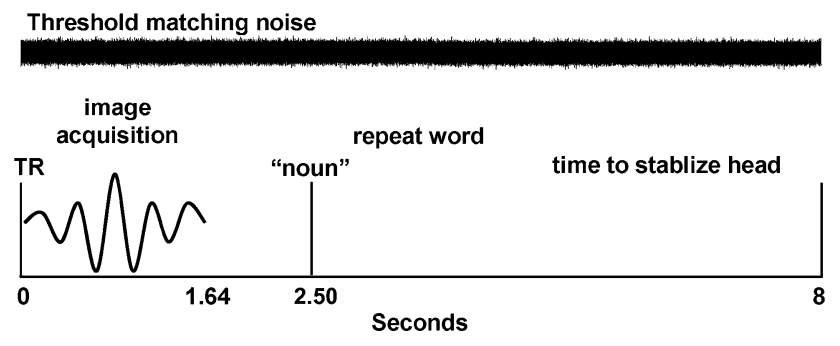

FIG. 1. Schematic of the experimental design used in this study for each trial. metric testing. A first level fixed-effects statistical analysis was performed for each individual's images to generate estimates of differences in activity for correct compared to incorrect word recognition. To avoid problems of multicollinearity that may have arisen from the dependency of subject performance on filter condition, separate first level fixed-effects analyses were performed to identify brain regions that parametrically varied across the four filter conditions. As described below, there was no age-related effect on word recognition. Therefore, all trials were included in the parametric filter condition analysis, which identified brain regions that were increasingly responsive to word intelligibility. In addition to the two dummy scans that were omitted for each run, the first real scan from each run was omitted to limit longitudinal magnetization effects that occur at the beginning of each fMRI experiment. The data were convolved with the SPM5 canonical hemodynamic response function and high-pass filtered at $128 \mathrm{~s}$.

Second level random-effects analyses were performed to examine age-related changes in brain regions engaged during correct versus incorrect responses, as well as age-related changes across the filter conditions. Based on the SPM results output, a joint statistical threshold of peak voxel $p<0.01$ and cluster extent $p<0.01$ was used for all of the second level analyses to be sensitive to sharp peak and broadly distributed effects (Poline et al. 1997). All of the peak voxel values reported in this study have probability values $<0.001$. A gray matter mask representing at least a $20 \%$ probability of gray matter across the sample, obtained from the subject's normalized and segmented gray matter images, was used to limit the analyses to gray matter regions and the number of statistical comparisons.

Voxel-based morphometry was performed using SPM5 to determine the extent to which the agerelated changes in brain activation could be attributed to structural declines. The T1-weighted images were normalized, segmented, bias field corrected, and modulated using an integrated generative model and the ICBM a priori gray matter, white matter, and CSF templates [unified segmentation (Ashburner and Friston 2005)]. The normalized, segmented, and modulated images were then smoothed using a 10 -mm kernel to ensure the data were normally distributed. A binary mask of the increasing intelligibility functional results was created to determine the extent to which speech responsive brain regions exhibited age-related declines in gray matter volume. The average voxel-wise gray matter volume, within the speech responsive regions associated with age, was collected using MarsBaR (Brett 2002). These values were used to determine the extent to which agerelated changes in left MFG activation were related to 
declining gray matter volume in speech responsive brain regions. An estimate of total gray matter volume was collected from the modulated and normalized gray matter images using custom Matlab (The Mathworks, Inc.) code (http://www.cs.ucl.ac.uk/staff/G. Ridgway/vbm/get_totals.m). This estimate of total gray matter volume was used in partial correlations to determine whether (1) specific age-related gray matter volume changes in speech responsive brain regions or (2) global declines in gray matter volume predicted age-related changes in left MFG activation described below.

\section{RESULTS}

Word recognition varied linearly with filter cut-off frequency ( $r=0.99$; Fig. 2A) and did not vary across

\section{A}

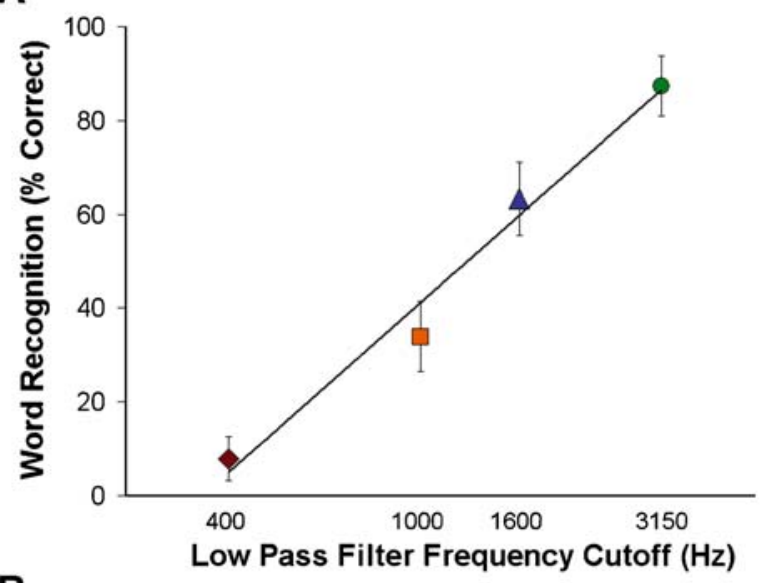

B

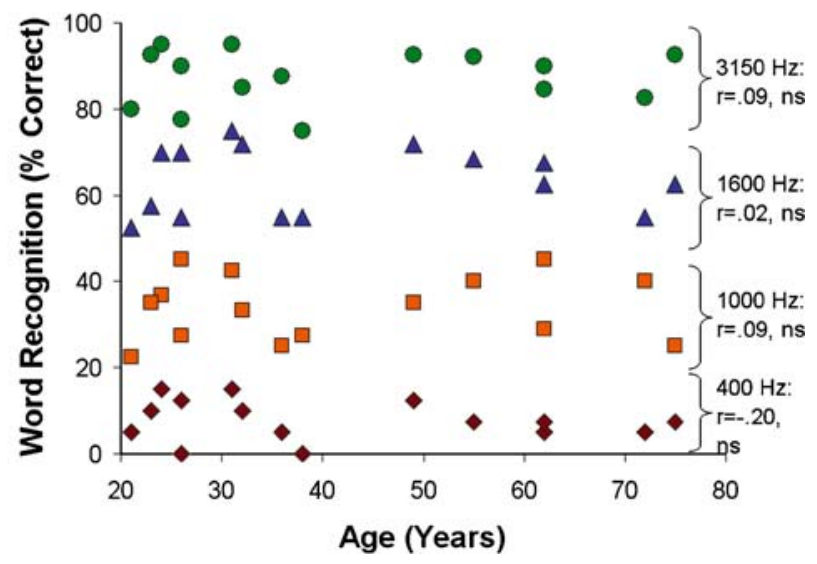

FIG. 2. Word recognition across the sample and by age. A Percentage of correct responses during the fMRI word recognition experiment. The filter cutoff frequencies were chosen to linearly affect word recognition based on preliminary pilot testing. Each colored symbol corresponds to each subject's performance for each filter condition in B. B There were no age-related changes in word recognition for any of the four filter conditions. age (Fig. 2B), indicating that word recognition was equivalent across age. In addition, there was no significant association between age and the percentage incorrect responses $(r=0.26$, ns) or "nope" responses $(r=-0.37, \mathrm{~ns})$. Across the subjects, increasing word intelligibility was associated with increasing activity in temporal lobe regions previously shown to be responsive to speech (Fig. 3; Binder et al. 2000; Obleser et al. 2006; Scott et al. 2006; Obleser et al. 2007). In particular, bilateral anterior superior temporal sulcus/superior temporal gyrus (STS/STG) and the left hippocampus/entorhinal cortex (HC) regions exhibited increasing activity with increasing word intelligibility.

Despite the absence of age-related changes in word recognition for any of the filter conditions, agerelated changes in brain activation were observed during word recognition. Older adults were more likely than younger adults to engage bilateral ACC, left MFG, bilateral calcarine, and left ventral occipital regions for correct compared to incorrect word recognition (Fig. 4, Supplemental Table 1). In addition, older adults were more likely to engage the left MFG in the most intelligible word condition $(3,150 \mathrm{~Hz})$ in comparison to the younger adults who were more likely to engage this region in the less intelligible word conditions (400, 1,000, and 1,600 Hz) (Supplemental Fig. 1). These results demonstrate that older adults increasingly engaged the left MFG in easy listening conditions associated with correct word recognition, but that younger adults increasingly engaged the left MFG in listening conditions that were likely to result in errors in word recognition.

In contrast to the age-related results for the left MFG, the entire sample demonstrated increased right frontal lobe activity for incorrect compared to correct word recognition and with increasingly filtered words (Supplemental Fig. 2A, B). In particular, there was

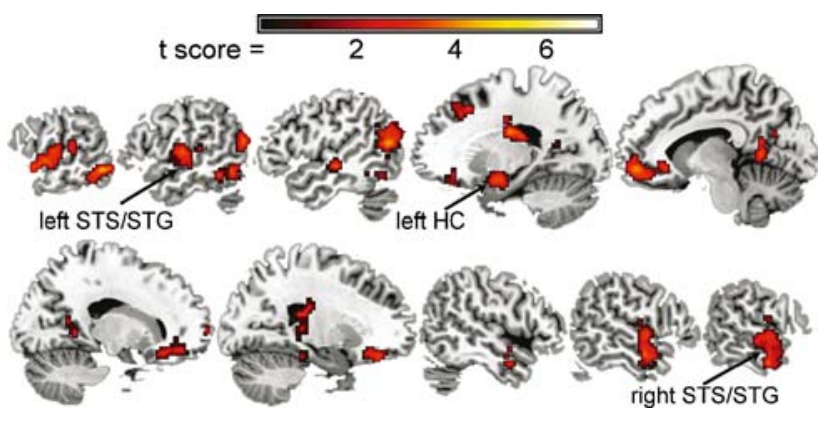

FIG. 3. Parametric increases in regional brain activity with increasing word intelligibility. These results, as well as the medial prefrontal and posterior cingulate regions, are consistent with the results of a previous study demonstrating increasing activity in these regions with increasing intelligibility for sentences that had a highly predictable semantic coherence (Obleser et al. 2007). 


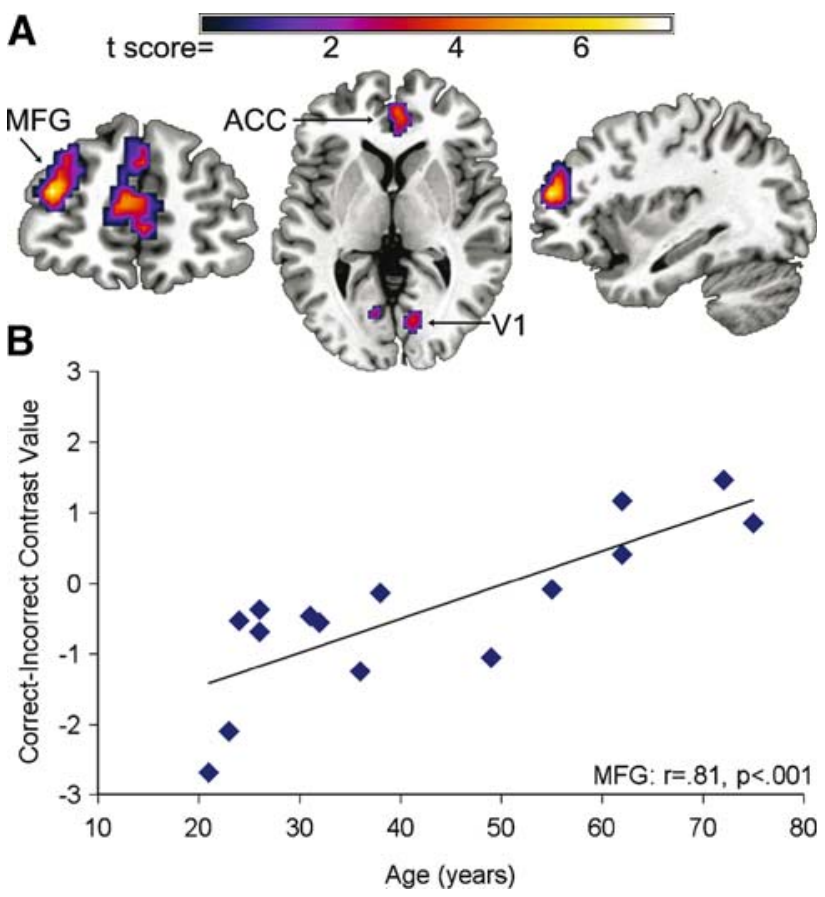

FIG. 4. Age-related increases in activity for correct compared to incorrect word recognition across the four filter conditions. A Cluster results demonstrating regions exhibiting age-related changes in left MFG, ACC, and visual cortex. B The results in A, and the left MFG in particular, occurred because older adults exhibited increased left MFG activity for correct word recognition, whereas younger adults exhibited increased left MFG activity for incorrect word recognition. The $y$-axis represents the average SPM contrast value from the MFG cluster.

increased right MFG and IFG activity for incorrect compared to correct word recognition and for the $400 \mathrm{~Hz}$ compared to $3,150 \mathrm{~Hz}$ filtered word conditions. Age was not associated with the contrast values from these right frontal regions (Supplemental Fig. 2C, D; Supplemental Tables 2 and 3).

Voxel-based morphometry correlation analyses demonstrated age-related declines within the gray matter regions exhibiting parametric increases in activity with increasing word intelligibility. Of the 7,394 voxels included in this analysis, significant agerelated declines in gray matter volume were observed in 15 left STS/STG voxels, 76 left HC voxels, and 48 left post-central sulcus/supramarginal gyrus voxels (SMG/PCS) (FDR $p<0.05$; Fig. 5A). Gray matter volume in these regions was also associated with the age-related changes in MFG activation for the correctincorrect comparison (Fig. 5B). In particular, the gray matter volume in the left STS/STG and left HC were significantly correlated with left MFG activation for the correct-incorrect and 400-3,150 Hz comparisons after controlling for total brain volume (Table 1). These results indicate that locally specific structural declines in the left STG/STS and left HC covary with the age-related functional changes in the left MFG.

\section{DISCUSSION}

Age-related changes in left MFG and ACC activity were observed during word recognition in clinically normal hearing adults. The age-related changes were dependent on listening difficulty, indicating that cognitive control systems are increasingly used with increasing age to make correct word recognition responses in easy listening conditions. While perception and memory studies demonstrate age-related increases in left MFG activity, the results of this study further indicate that age-related structural declines in speech-responsive temporal lobe regions are tightly correlated with the increased left MFG activity. These results suggest that declining structural integrity of temporal lobe regions that support speech recognition leads to increased reliance on cognitive control systems to recognize words.

Our interpretation that greater cognitive control is required for the easiest word recognition conditions with increasing age is consistent with the age-related gray matter volume declines in temporal lobe regions that were responsive to increasing word intelligibility. Declining structural integrity of the left STS/STG and left $\mathrm{HC}$ predicted the age-related increase in reliance on left MFG activity for word recognition, even after controlling for global declines in gray matter volume.

A
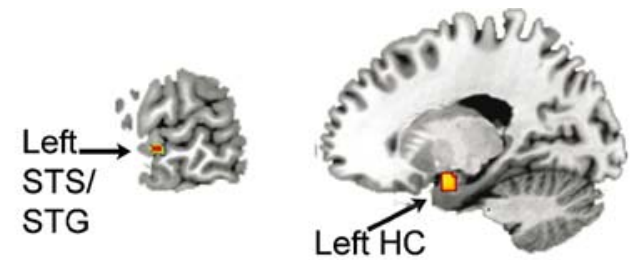

B

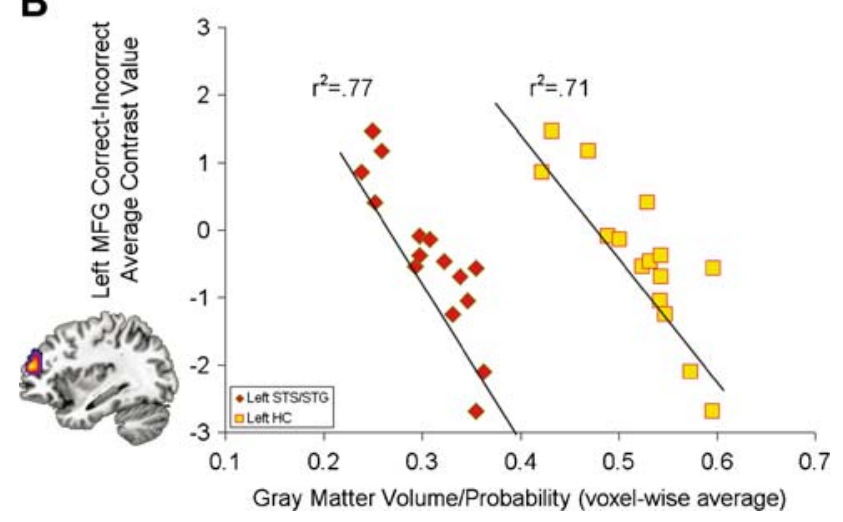

FIG. 5. Age-related changes in gray matter volume predict left MFG activation. A Age-related changes in left STS/STG $(-64,-10$, and -4$)$ and left $\mathrm{HC}(-18,0$, and -16$)$ regions that were responsive to increasing speech intelligibility and predicted left MFG activation during word recognition, after controlling for brain volume. The left SMG/PCS $(-42,-40$, and 54; not shown) was not significantly predictive of left MFG activation after controlling for brain volume. B Gray matter volume in the left STS/STG (red diamonds) and left HC (yellow squares) predict left MFG activation from the correctincorrect comparison (shown on the $y$-axis). 
TABLE 1

Gray matter volume in speech responsive regions predicts age-related changes in left MFG activation during word recognition



This result indicates that people with declining structural integrity of speech-responsive brain regions rely on cognitive control systems to perform word recognition tasks. In contrast to the older adults in this study, older adults with speech recognition difficulties may not be capable of relying on frontal lobe systems to compensate for degraded speech representations (Tremblay et al. 2002). Impairments in cognitive control may also explain why many older adults experience dissatisfaction and limited benefit from hearing aids.

Age-related changes in ACC and MFG activation have been observed for perceptual tasks (Grady et al. 1994; Fernandes et al. 2006; Moffat et al. 2006), such as the face and spatial-location matching, as well as memory (Cabeza et al. 1997; Grady et al. 1999; McIntosh et al. 1999; Reuter-Lorenz et al. 2000; Rypma and D'Esposito 2000; Cabeza et al. 2002; Grady et al. 2006; Grady et al. 2007) and response inhibition tasks (Milham et al. 2002; Nielson et al. 2002). We have interpreted the findings of this study as reflecting age-related changes in cognitive control, which is consistent with functional roles attributed to the MFG. Cognitive control is a broad construct, however, and could include response selection and suppression, directing attention, performance monitoring, or encoding and memory retrieval.

The age-related changes in ACC activation suggest that participants were engaging a system consistently shown to be important for conflict monitoring and error detection. In particular, the ACC is hypothesized to provide MFG with information about conflicting or ambiguous perceptual information so that MFG can guide the selection of any appropriate response (Botvinick et al. 1999; Kerns et al. 2004; Ridderinkhof et al. 2004). Age-related increases in cognitive control for the easiest listening condition would result in an up-regulation of the ACC, as well as the MFG. This interpretation is consistent with evidence for age-related changes in ACC during speech comprehension (Sharp et al. 2006). In this context, older adults may be monitoring their perfor- mance to a greater extent than younger adults in the easiest listening conditions while younger adults monitor performance to a greater extent in the more difficult listening conditions.

Increased task difficulty could also lead to an upregulation of conflict monitoring systems. ACC and MFG regions are increasingly engaged with increasing task difficulty (Barch et al. 1997; Mattay et al. 2006; Tregellas et al. 2006). Age-related changes may be observed in these regions because relatively easy tasks may be more challenging for older adults compared to younger adults. For example, Grady et al. (1994) demonstrated age-related increases in left MFG activity for face matching and spatial-location matching tasks. These age-related changes appeared to be diminished for face matching and spatial-location matching tasks that required longer reaction times, suggesting they were more difficult. One strength of our parametric design was that it demonstrated agerelated changes in left MFG activity with decreasing word intelligibility. Older adults in the sample demonstrated increased left MFG activity for the easiest listening condition while younger adults in the sample demonstrated comparatively increased left MFG activity for the most difficult conditions. This result is important because it indicates that age-related changes in blood oxygen level-dependent signal vary depending on the difficulty of the cognitive task. Similar observations have been reported from memory experiments in which older adults exhibit greater activity in MFG regions during relatively easier memory load conditions while younger adults exhibit increased activity in these regions with increasing memory load (Mattay et al. 2006). These results have been interpreted as a decline in neural efficiency that represents a need for recruitment of additional resources in relatively easy task conditions (ReuterLorenz 2002; Mattay et al. 2006).

An alternative explanation for our age-related MFG results is that a short-term memory strategy was 
differentially used across age to perform the word recognition task. Older adults often fail to inhibit irrelevant or extraneous information, which has been associated with a richer array of information in working memory compared to younger adults (Hasher and May 1999). The age-related changes in the left MFG may reflect the engagement of frontal memory systems for previously presented words or a refreshing of representation for words in left MFG (Brodmann area 10, 46; Johnson et al. 2005). The association between increasing age and activation in posterior STG/STS regions implicated in phonological working memory (Hickok et al. 2000; Buchsbaum et al. 2005) for the 3,150-400 Hz comparison (Supplementary Fig. 1) supports the interpretation that short-term memory systems are increasingly engaged in easy listening conditions with increasing age. In addition, declines in hippocampal gray matter volume, within regions engaged by the word recognition task, were significantly correlated with left MFG activation. This observation is consistent with evidence of age-related increases in correlated activity between hippocampal and left MFG regions during memory encoding (Springer et al. 2005). Declining hippocampal integrity may increase the reliance on left MFG regions for the simplest of perceptual and memory tasks.

The results of this study indicate that increasing engagement of left MFG during word recognition begins in middle age, given the age range of our subjects. Importantly, this age-related change in activity was tightly correlated with declines in gray matter volume in regions that support word recognition and memory. Declining structural integrity of speech-responsive brain regions appears to result in a reliance on frontal lobe cognitive control systems to recognize speech in easy listening conditions. These results are consistent with a large body of evidence that older adults rely on prefrontal cortex for memory and perceptual tasks and for the first time directly implicates structural decline in the hippocampus and anterior STG, regions consistently engaged in memory and speech recognition tasks. We hypothesize that the increased need for cognitive control for successful word recognition is the basis for fatigue that many older adults with hearing loss experience during normal conversation and that perturbation of this cognitive control system results in a failure to inhibit competing sensory stimuli and impaired speech recognition.

\section{ACKNOWLEDGEMENTS}

We would like to thank the participants of this study, Jillanne Schulte for her help with pilot testing the word recognition experiment, the NIDCD (P50 DC00422), and the MUSC
Center for Advanced Imaging Research. This investigation was conducted in a facility constructed with support from Research Facilities Improvement Program Grant Number C06 RR14516 from the National Center for Research Resources, National Institutes of Health. This research was conducted while Mark Eckert was an AFAR Research Grant recipient.

\section{REFERENCES}

Ansi. Specification for Audiometrics. New York, American National Standards Institute, 2004.

Ashburner J, Friston KJ. Unified segmentation. Neuroimage 26:839-851, 2005.

Barch DM, Braver TS, Nystrom LE, Forman SD, Noll DC, Cohen JD. Dissociating working memory from task difficulty in human prefrontal cortex. Neuropsychologia 35:1373-1380, 1997.

Binder JR, Frost JA, Hammeke TA, Bellgowan PS, Springer JA, Kaufman JN, Possing ET. Human temporal lobe activation by speech and nonspeech sounds. Cereb. Cortex 10:512-528, 2000.

Botvinick M, Nystrom LE, Fissell K, Carter CS, Cohen JD. Conflict monitoring versus selection-for-action in anterior cingulate cortex. Nature 402:179-181, 1999.

Brett M, Anton J, Valabregue, R, Poline J. Region of interest analysis using an SPM toolbox. 8th International Conference on Functional Mapping of the Human Brain, Sendai, Japan, 2002.

Buchsbaum BR, Olsen RK, Koch P, Berman KF. Human dorsal and ventral auditory streams subserve rehearsal-based and echoic processes during verbal working memory. Neuron 48:687-697, 2005.

Cabeza R, Grady CL, Nyberg L, McIntosh AR, Tulving E, Kapur S, Jennings JM, Houle S, Craik FI. Age-related differences in neural activity during memory encoding and retrieval: a positron emission tomography study. J. Neurosci. 17:391-400, 1997.

Cabeza R, Anderson ND, Locantore JK, McIntosh AR. Aging gracefully: compensatory brain activity in high-performing older adults. Neuroimage 17:1394-1402, 2002.

Cabeza R, Daselaar SM, Dolcos F, Prince SE, Budde M, Nyberg L. Task-independent and task-specific age effects on brain activity during working memory, visual attention and episodic retrieval. Cereb. Cortex 14:364-375, 2004.

Colcombe SJ, Kramer AF, Erickson KI, Scalf P. The implications of cortical recruitment and brain morphology for individual differences in inhibitory function in aging humans. Psychol. Aging 20:363-375, 2005.

Dirks DD, Takayanagi S, Moshfegh A. Effects of lexical factors on word recognition among normal-hearing and hearing-impaired listeners. J. Am. Acad. Audiol. 12:233-244, 2001.

Dubno JR, Lee FS, Matthews LJ, Mills JH. Age-related and genderrelated changes in monaural speech recognition. J. Speech Lang. Hear. Res. 40:444-452, 1997.

Dubno JR, Horwitz AR, Ahlstrom JB. Recognition of filtered words in noise at higher-than-normal levels: decreases in scores with and without increases in masking. J. Acoust. Soc. Am. 118:923-933, 2005.

Dubno JR, Horwitz AR, Ahlstrom JB. Spectral and threshold effects on recognition of speech at higher-than-normal levels. J. Acoust. Soc. Am. 120:310-320, 2006.

Dywan J, Segalowitz SJ, Webster L, Hendry K, Harding J. Eventrelated potential evidence for age-related differences in attentional allocation during a source monitoring task. Dev. Neuropsychol. 19:99-120, 2001.

Dywan J, Segalowitz S, Arsenault A. Electrophysiological response during source memory decisions in older and younger adults. Brain Cogn. 49:322-340, 2002.

Fernandes MA, Pacurar A, Moscovitch M, Grady C. Neural correlates of auditory recognition under full and divided 
attention in younger and older adults. Neuropsychologia 44:2452-2464, 2006.

Fridriksson J, Morrow KL, Moser D, Baylis GC. Age-related variability in cortical activity during language processing. $\mathrm{J}$. Speech Lang. Hear. Res. 49:690-697, 2006.

GazZAley A, D'Esposito M. Top-down modulation and normal aging. Ann. N. Y. Acad. Sci. 1097:67-83, 2007.

Gordon-Salant S, Fitzgibbons PJ. Sources of age-related recognition difficulty for time-compressed speech. J. Speech Lang. Hear. Res. 44:709-719, 2001.

Gordon-Salant S, Fitzgibbons PJ. Effects of stimulus and noise rate variability on speech perception by younger and older adults. J. Acoust. Soc. Am. 115:1808-1817, 2004.

Gracco VL, Tremblay P, Pike B. Imaging speech production using fMRI. Neuroimage 26:294-301, 2005.

Grady Cl, Maisog JM, Horwitz B, Ungerleider LG, Mentis MJ, Salerno JA, Pietrini P, Wagner E, Haxby JV. Age-related changes in cortical blood flow activation during visual processing of faces and location. J. Neurosci. 14:1450-1462, 1994.

Grady CL, Mcintosh AR, Rajah MN, Beig S, Craik FI. The effects of age on the neural correlates of episodic encoding. Cereb. Cortex 9:805-814, 1999.

Grady CL, MCIntosh AR, CRAIK FI. Task-related activity in prefrontal cortex and its relation to recognition memory performance in young and old adults. Neuropsychologia 43:1466-1481, 2005.

Grady CL, Springer MV, Hongwanishrul D, McIntosh AR, Winocur G. Age-related changes in brain activity across the adult lifespan. J. Cogn. Neurosci. 18:227-241, 2006.

Grady CL, Yu H, Alain C. Age-related differences in brain activity underlying working memory for spatial and nonspatial auditory information. Cereb. Cortex 18:189-199, 2007.

Hasher LZR, May CP. Inhibitory Control, Circadian Arousal, and Age. In: Gopher D, Koriat A (eds) Attention and Performance XVII. Cambridge, MA, MIT Press, pp. 653-675, 1999.

Hickok G, Erhard P, Kassubek J, Helms-Tillery AK, Naeve-Velguth S, Strupp JP, STRICK PL, Ugurbil K. A functional magnetic resonance imaging study of the role of left posterior superior temporal gyrus in speech production: implications for the explanation of conduction aphasia. Neurosci. Lett. 287:156-160, 2000.

Johnson MK, Raye CL, Mitchell KJ, Greene EJ, Cunningham WA, SANISLOW CA. Using fMRI to investigate a component process of reflection: prefrontal correlates of refreshing a just-activated representation. Cogn. Affect Behav. Neurosci. 5:339-361, 2005.

Kerns JG, Cohen JD, MacDonald AW, 3Rd, Cho RY, Stenger VA, CARTER CS. Anterior cingulate conflict monitoring and adjustments in control. Science 303:1023-1026, 2004.

Mattay VS, Fera F, Tessitore A, Hariri AR, Berman KF, Das S, MeyerLindenberg A, Goldberg TE, Callicott JH, Weinberger DR. Neurophysiological correlates of age-related changes in working memory capacity. Neurosci. Lett. 392:32-37, 2006.

Mcintosh AR, Sekuler AB, Penpeci C, Rajah MN, Grady CL, Sekuler R, BennetT PJ. Recruitment of unique neural systems to support visual memory in normal aging. Curr. Biol. 9:1275-1278, 1999.

Milham MP, Erickson KI, Banich MT, Kramer AF, Webb A, Wszalek T, CoHEN NJ. Attentional control in the aging brain: insights from an fMRI study of the Stroop task. Brain Cogn. 49:277-296, 2002.

Moffat SD, Elkins W, Resnick SM. Age differences in the neural systems supporting human allocentric spatial navigation. Neurobiol. Aging 27:965-972, 2006.
Nielson KA, Langenecker SA, Garavan H. Differences in the functional neuroanatomy of inhibitory control across the adult life span. Psychol. Aging 17:56-71, 2002.

Obleser J, Boecker H, Drzezga A, Haslinger B, Hennenlotter A, Roettinger M, Eulitz C, Rauschecker JP. Vowel sound extraction in anterior superior temporal cortex. Hum. Brain Mapp. 27:562-571, 2006.

Obleser J, Wise RJ, Alex Dresner M, Scott SK. Functional integration across brain regions improves speech perception under adverse listening conditions. J. Neurosci. 27:2283-2289, 2007.

Poline JB, Worsley KJ, Evans AC, Friston KJ. Combining spatial extent and peak intensity to test for activations in functional imaging. Neuroimage 5:83-96, 1997.

Raz N, Gunning FM, Head D, Dupuis JH, McQuain J, Briggs SD, LOKEN WJ, ThORNTON AE, ACKER JD. Selective aging of the human cerebral cortex observed in vivo: differential vulnerability of the prefrontal gray matter. Cereb. Cortex 7:268-282, 1997.

Reuter-Lorenz PA, Jonides J, Smith Ee, Hartley A, Miller A, Marshuetz C, Koeppe RA. Age differences in the frontal lateralization of verbal and spatial working memory revealed by PET. J. Cogn. Neurosci. 12:174-187, 2000.

Reuter-Lorenz P. New visions of the aging mind and brain. Trends Cogn Sci 6:394-400, 2002.

Ridderinkhof KR, Ullsperger M, Crone EA, Nieuwenhuis S. The role of the medial frontal cortex in cognitive control. Science 306:443-447, 2004.

Rypma B, D'Esposito M. Isolating the neural mechanisms of agerelated changes in human working memory. Nat. Neurosci. 3:509-515, 2000.

ScotT SK, Rosen S, LANG H, WISE RJ. Neural correlates of intelligibility in speech investigated with noise vocoded speech - a positron emission tomography study. J. Acoust. Soc. Am. 120:1075-1083, 2006.

Sharp DJ, Scott SK, Mehta MA, Wise RJ. The neural correlates of declining performance with age: evidence for age-related changes in cognitive control. Cereb. Cortex 16:1739-1749, 2006.

Shuster LI, Lemieux SK. An fMRI investigation of covertly and overtly produced mono- and multisyllabic words. Brain Lang. 93:20-31, 2005.

SOMMERs MS. Stimulus variability and spoken word recognition. II. The effects of age and hearing impairment. J. Acoust. Soc. Am. 101:2278-2288, 1997.

SOMmers MS, DANIELSON SM. Inhibitory processes and spoken word recognition in young and older adults: the interaction of lexical competition and semantic context. Psychol. Aging 14:458-472, 1999.

Springer MV, McIntosh AR, Winocur G, Grady CL. The relation between brain activity during memory tasks and years of education in young and older adults. Neuropsychology 19:181-192, 2005.

Tisserand DJ, van Boxtel MP, Pruessner JC, Hofman P, Evans AC, Jolles J. A voxel-based morphometric study to determine individual differences in gray matter density associated with age and cognitive change over time. Cereb. Cortex 14:966-973, 2004.

Tregellas JR, Davalos DB, Rojas DC. Effect of task difficulty on the functional anatomy of temporal processing. Neuroimage 32:307-315, 2006.

Tremblay KL, Piskosz M, Souza P. Aging alters the neural representation of speech cues. Neuroreport 13:1865-1870, 2002.

van Rooij JC, Plomp R. Auditive and cognitive factors in speech perception by elderly listeners. II: Multivariate analyses. J. Acoust. Soc. Am. 88:2611-2624, 1990. 